Classification

Physics Abstracts

0.620

\title{
RÉALISATION D'UN THERMOSTAT POUR CELLULE DE MESURE DESTINÉE A LA SPECTROMÉTRIE DE LUMIÈRE LASER DIFFUSÉE PAR DES PARTICULES EN SUSPENSION
}

\author{
A. BOUILLER et H, RIDELAIRE \\ Laboratoire de Physique Electronique, Université Libre de Bruxelles \\ 50, avenue Franklin-Roosevelt, 1050 Bruxelles, Belgique
}

(Reçu le 4 avril 1975, révisé le 16 mai 1975, accepté le 29 mai 1975)

\begin{abstract}
Résumé. - Les expériences de lumière laser diffusée sur des systèmes fluides nécessitent une bonne stabilité en température de l'échantillon, non seulement pour obtenir un contrôle précis de la température, mais aussi pour éviter les courants de convection dans le fluide pendant les mesures. Nous présentons une description du thermostat qui est réalisé pour effectuer des expériences au-dessus de la température ambiante. L'appareil présente les avantages suivants : la régulation de température est réalisée sans fluide de refroidissement et fournit une précision de $0,02{ }^{\circ} \mathrm{C}$ entre $27^{\circ} \mathrm{C}$ et $33{ }^{\circ} \mathrm{C}$; l'absence de fenêtres optiques permet les mesures à tous les angles de diffusion sans correction de chemin optique due aux fenêtres. Ce type de thermostat apparaît comme particulièrement approprié pour les études de lumière diffusée sur les suspensions colloïdales et les microorganismes vivants.
\end{abstract}

Abstract. - Laser light scattering experiments on fluid systems require a good temperature stability of the sample, not only to achieve an accurate control of temperature, but also to avoid convection currents in the fluid during measurements. We present a description of the thermostat which allows one to perform experiments above room temperature. The apparatus exhibits the following advantages : the temperature regulation is maintained without cooling the fluid system and provides an accuracy of $0.02{ }^{\circ} \mathrm{C}$ between $27^{\circ} \mathrm{C}$ and $33^{\circ} \mathrm{C}$; the absence of optical windows allows measurements at all scattering angles without optical paths corrections due to such windows. This type of thermostat appears to be particularly appropriate for light scattering studies of colloïdal suspensions and living microorganisms.

Le but poursuivi en construisant ce thermostat est la régulation à $\pm 0,05^{\circ} \mathrm{C}$ près de la température d'une enceinte dans la gamme de 27 à $35^{\circ} \mathrm{C}$. Nous désirions nous assurer de la constance au millième près de la viscosité du méthanol dans lequel sont mises en suspension des billes de polystyrène de $0,176 \mu \mathrm{m}$ de diamètre. Cette suspension devait être utilisée pour une étude du mouvement brownien de particules dans un fluide par relevé de la fonction d'autocorrélation des photons diffusés.

1. Description du thermostat. - Le thermostat (Fig. 1) se compose d'un bloc en laiton cylindrique de $82 \mathrm{~mm}$ de diamètre et de $103 \mathrm{~mm}$ de hauteur, évidé en son centre par un trou de $27 \mathrm{~mm}$ de diamètre et de $56 \mathrm{~mm}$ de profondeur dans lequel doit être placée la cellule de mesure. Vingt quatre petites chambres sont réparties circulairement dans le bloc, chacune recevant un élément chauffant. Ces éléments sont vissés d'une part dans le bloc qui constitue un point d'alimentation

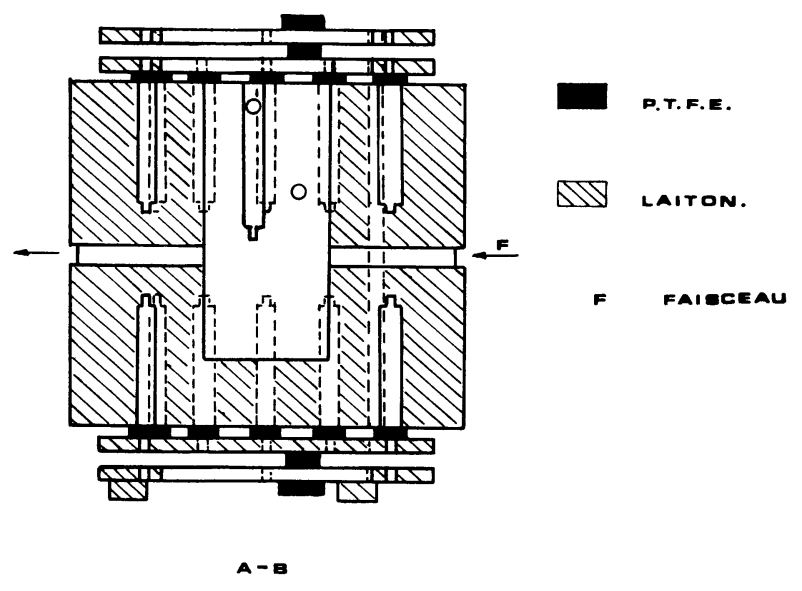

FIG. 1. - Vue en plan du bloc régulateur.

électrique, d'autre part soudés sur quatre couronnes en laiton isolées l'une de l'autre deux à deux, constituant le deuxième point d'alimentation. A mi-hauteur, une fente transversale de $4 \mathrm{~mm}$ de hauteur a été fraisée 
sur un peu plus de la moitié du cylindre (Fig. 1 et 2). Elle laisse passer le faisceau laser suivant BA à travers la cellule de diffusion centrée dans le thermostat, et permet la détection de la lumière diffusée suivant un angle choisi entre $0^{\circ}$ et $180^{\circ}$ dans la direction de la face ADB (Fig. 2).
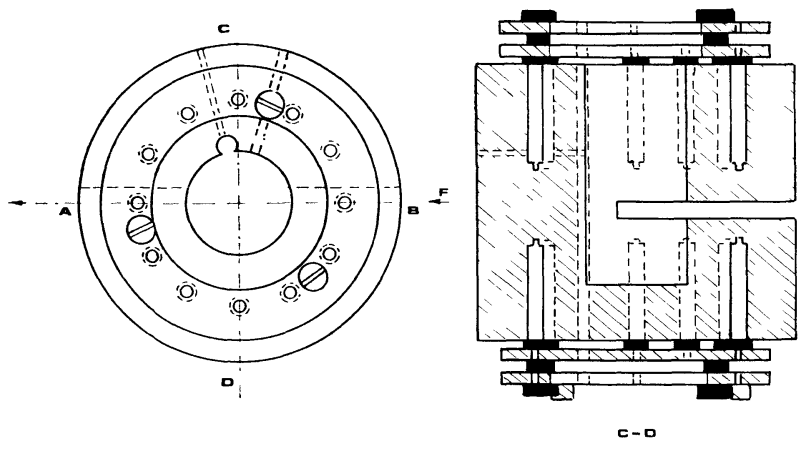

FIG. 2. - Vue de profil du bloc régulateur.

L'élément détecteur destiné à la régulation de température a été placé dans une cavité percée orthogonalement à l'axe du cylindre, près du diamètre $C D$ (Fig. 2). Le thermomètre de platine pour la mesure de la température, est fixé verticalement le long de la paroi interne du cylindre (Fig. 1). L'enceinte est centrée dans un support isolant en résine époxy, type araldite, et isolée thermiquement de l'extérieur par des parois en polystyrène; l'ensemble est encastré dans un boîtier d'aluminium $(1 \mathrm{~cm}$ d'épaisseur), lui-même centré sur une table tournante permettant les changements d'angle de détection (Fig. 3). Un miroir orientable coulisse le long d'un bras solidaire de la table tournante; il est réglé pour réfléchir le faisceau laser

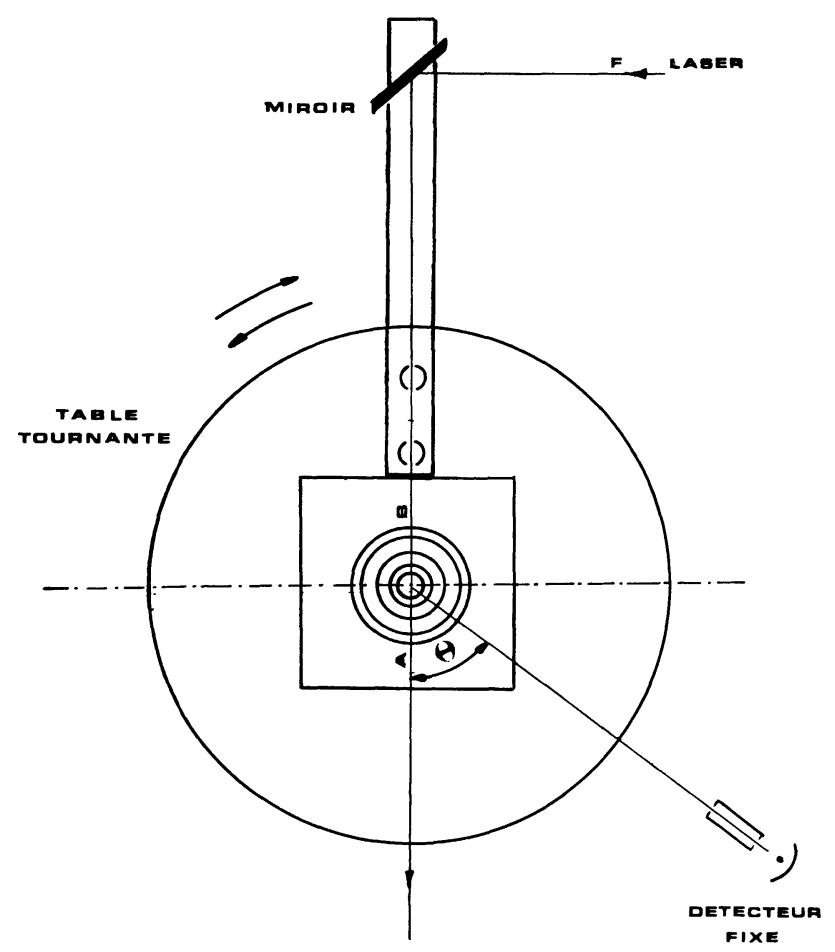

FIG. 3. - Schéma des trajets optiques. suivant l'axe diamétral $\mathrm{AB}$. Un alignement optique préalable est nécessaire avant la mise en place du thermostat contenant la cellule de mesure. La détection de la lumière diffusée se fait suivant un axe diamétral de la table, à travers une direction d'alignement fixe, délimitée par des trous d'épingle. Celle-ci forme avec l'axe du faisceau incident (BA) un angle $\theta$ variant de $0^{\circ}$ à $180^{\circ}$. Une tige en plexiglass supporte le bouchon de l'enceinte en laiton et traverse le couvercle du boîtier d'aluminium permettant d'orienter, si nécessaire, la cellule dans le thermostat (Fig. 4). Nous avons utilisé

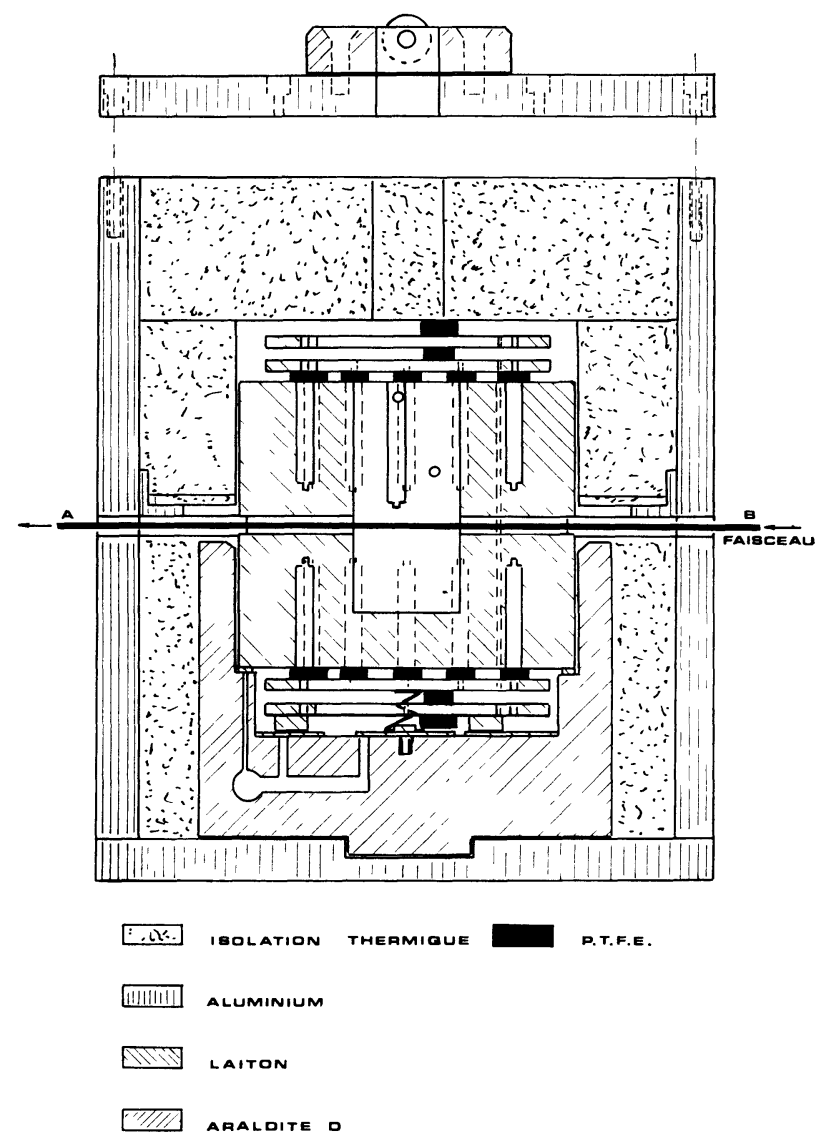

Fig. 4. - Vue en plan du thermostat.

deux types de cellules optiques à axe vertical : l'une cylindrique (de $23 \mathrm{~mm}$ de diamètre), l'autre rectangulaire (Helma 101). Les seules corrections de trajet optique effectuées sont relatives au passage à travers les parois de la cellule de diffusion.

2. Régulation. - La détection de la variation de température se fait à partir de la variation de résistivité d'un élément résistant «C. T. N. miniature » de $100 \mathrm{k} \Omega$ à $20^{\circ} \mathrm{C}$, dont le coefficient de température entre 25 et $30^{\circ} \mathrm{C}$ est de l'ordre de $200 \Omega$ par $0,1^{\circ} \mathrm{C}$.

La «C.T. N.» et un potentiomètre multi-tours constituent une branche d'un pont de Wheatstone dont l'autre est composée de résistances fixes, bobinées, ayant un faible coefficient de température de $3 \times 10^{-6} \Omega$ pour $0,1^{\circ} \mathrm{C}$. Nous choisissons quatre résistances de valeurs à peu près égales, alimentées par une source à 
basse impédance de façon à obtenir la plus grande sensibilité du pont (P. Baudoux [1]). La constante de dissipation de la «C. T. N. " étant de $0,4 \mathrm{~mW} /{ }^{\circ} \mathrm{C}$ à 1 'air libre, il convenait d'alimenter le pont sous faible différence de potentiel pour ne pas provoquer d'échauffement de la «C. T. N. » par le courant qui la traverse. Nous avons choisi 5 piles en série de 1,5 V (Fig. 5).

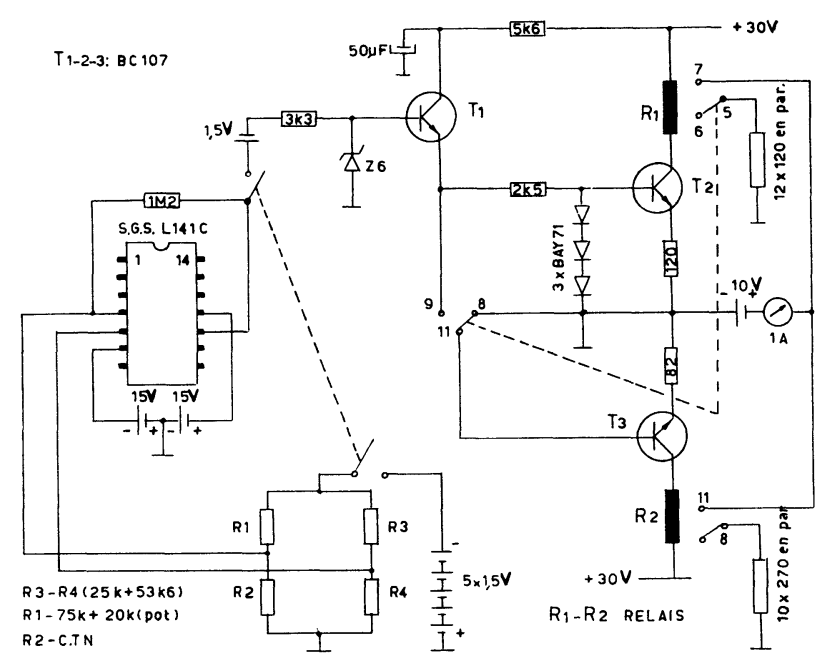

Fig. 5. - Schéma des circuits électroniques de régulation.

La tension de déséquilibre du pont $\delta v_{0}$ pour une variation $\delta R$ de la thermistance C. T. N. $R_{\text {th }}$ autour de la valeur correspondant à une température désirée, s'exprime par la relation (P. Baudoux [1])

$$
\delta v_{0}=\frac{\delta R}{R^{2}} \cdot \frac{R_{\mathrm{A}} V}{\left(\sqrt{\left.\frac{R_{\mathrm{A}}}{R}+\sqrt{\frac{R_{\mathrm{A}}}{R}+1}\right)^{2}}\right.}
$$

où $R_{\mathrm{A}}$ est la résistance interne du détecteur (haute impédance d'entrée d'un amplificateur opérationnel); $R$ est la valeur des résistances du pont à l'équilibre $(100 \mathrm{k} \Omega)$.

Dans notre cas $R_{\mathrm{A}} \gg R$.

$$
\delta v_{0} \simeq \frac{\delta R}{4 R} V
$$

soit $\delta v_{0}=3,75 \mathrm{mV}$ pour $0,1^{\circ} \mathrm{C}$.

Cette valeur de la tension de déséquilibre sert de base pour la commande de la régulation en température du thermostat. Le choix du détecteur s'est porté sur l'amplificateur opérationnel de référence L $141 \mathrm{C}$, qui se caractérise par un gain élevé en boucle ouverte, une grande impédance à l'entrée et la symétrie de celle-ci. Le signal à la sortie du détecteur doit être suffisamment élevé $(\sim 1 \mathrm{~V})$ pour rendre conducteur le transistor $\mathrm{T}_{1}$ normalement bloqué (Fig. 5). Le gain $G$ de l'amplificateur opérationnel qui détermine la résistance de contre-réaction $R_{\mathrm{CR}}$ à utiliser pour obtenir $1 \mathrm{~V}$ à sa sortie $\left(V_{\mathrm{s}}\right)$, est donné par l'expression :

$$
G=\frac{V_{\mathrm{s}}}{V_{\mathrm{e}}}=275
$$

soit :

$$
G=\frac{-R_{\mathrm{CR}}}{R_{\mathrm{E}}} \quad(\mathrm{R} . \text { Damaye }-[2])
$$

où $R_{\mathrm{E}}$ est la résistance équivalente :

$$
R_{\mathrm{E}}=\frac{R_{1} \cdot R_{\mathrm{th}}}{R_{1}+R_{\mathrm{th}}}
$$

$R_{\mathrm{E}}=50 \mathrm{k} \Omega$ donc $R_{\mathrm{CR}}=1,375 \times 10^{7} \Omega$.

Le fait d'insérer une résistance aussi élevée diminue le rapport signal/bruit en augmentant la sensibilité du montage aux inductions parasites; aussi, nous avons préféré diviser par 10 la valeur de $\boldsymbol{R}_{\mathrm{CR}}$ et intercaler en série avec la sortie de l'amplificateur une pile de $1,5 \mathrm{~V}$, ce qui a pour effet de déplacer le seuil. Dans ces conditions, à l'équilibre du pont, le transistor $T_{1}$ (Fig. 5) est bloqué tandis que, pour un $\Delta V_{0}$ correspondant à $0,05^{\circ} \mathrm{C}$, il conduit. La diode Zener $\mathrm{Z} 6$ dans la base de $\mathrm{T}_{1}$ limite le potentiel de celle-ci à $6 \mathrm{~V}$ lors d'un important déséquilibre du pont. Par l'émetteur de $T_{1}$, le signal est appliqué à la base de $T_{2}$, à travers une résistance de $2,5 \mathrm{k} \Omega$; trois diodes BAY 71 fixent la tension base de $\mathrm{T}_{2}$ approximativement à $2,5 \mathrm{~V}$. La résistance d'émetteur de $T_{2}$ est choisie en fonction de la valeur du courant de maintien du relais inverseur $R_{1}$, qui commande le premier circuit de préchauffage dissipant une puissance de $10 \mathrm{~W}$. Au fur et à mesure que l'on se rapproche de l'équilibre du pont, la tension base diminue, et en dessous de $2,5 \mathrm{~V}$ le courant dans le transistor devient insuffisant. Le relais $R_{1}$ bascule et met alors en circuit le transistor $T_{3}$. Celui-ci était bloqué, sa base étant à la masse par les contacts de $R_{1}$. $T_{3}$ reçoit le signal de l'émetteur de $T_{1}$. Un second relais $R_{2}$ dans le circuit collecteur de $T_{3}$ commande le deuxième circuit de chauffage (d'entretien) dissipant 2,5 W. Lorsque le pont atteint l'équilibre, le courant émetteur de $\mathrm{T}_{3}$ ne peut plus maintenir l'enclenchement du relais et le deuxième circuit de chauffage est coupé. A cause des pertes de chaleur, dues principalement à l'existence de la fente d'observation, la masse de laiton se refroidit, provoquant un déséquilibre du pont ; un signal réapparaît à la base de $T_{3}$ qui enclenche le relais $R_{2}$ et ferme le circuit de chauffage d'entretien, puis le cycle recommence.

Avec ce type de régulation, la température dans le thermostat varie de $\pm 0,02^{\circ} \mathrm{C}$. Pour disposer d'un affichage direct de la température du thermostat, nous avons procédé à un étalonnage du potentiomètre en fonction des valeurs de la résistance C.T.N.

3. Mesure de la température. - La mesure de la température dans l'enceinte est faite par un thermomètre constitué d'une résistance de platine de $50 \Omega$ à $0^{\circ} \mathrm{C}$ (construite suivant les normes DIN 43760). Nous avons choisi ce type de thermomètre à cause de sa bonne stabilité au cours du temps, ainsi que de sa linéarité de réponse dans la gamme limitée des températures envisagées (McCullough [3]). Nous avons procédé à l'étalonnage simultané de cette résistance et 
Valeur affichée au potentiomètre

$\begin{array}{rll}- & -\overline{3} & - \\ 0 & 55,33 \Omega & 55,33 \Omega \\ 100 & 55,43 & 55,44 \\ 200 & 55,55 & 55,55 \\ 300 & 55,65 & 55,66 \\ 400 & 55,78 & 55,78 \\ 500 & 55,90 & 55,90 \\ 600 & 56,01 & 56,02 \\ 700 & 56,15 & 56,15 \\ 800 & 56,28 & 56,28 \\ 900 & 56,41 & 56,41 \\ 1000 & 56,56 & 56,55\end{array}$

de la thermistance (C. T. N.) dans un calorimètre approprié.

Les deux résistances, ainsi qu'un thermomètre à mercure de référence gradué à $1 / 10^{\circ} \mathrm{C}$ pour $1 \mathrm{~mm}$, sont introduits simultanément dans un bloc de laiton placé dans un thermostat à circulation d'eau. Pour augmenter la conductibilité thermique entre les trois éléments placés dans ce bloc de laiton, nous les avons enrobés de graisse silicone. Au moyen d'une loupe, la lecture des graduations du thermomètre est faite à mieux que $0,25 \mathrm{~mm}$, ce qui correspond à une précision absolue sur la lecture de la température inférieure à $0,03^{\circ} \mathrm{C}$. Nous nous sommes fixés six points de régulation du calorimètre entre $24^{\circ}$ et $35^{\circ} \mathrm{C}$, et nous avons effectué à chacun d'eux la mesure de la résistance de platine et celle de l'élément $C$. T. N. Cette mesure est faite au pont Mueller (Leeds et Northrup) dont la sensibilité est de $10^{-4} \Omega$. Nous avons exploité la linéarité de réponse de la résistance de platine $R_{\mathrm{Pt}}$, en traçant la droite $R_{\mathrm{Pt}}=A t$, passant au mieux par les 6 valeurs de résistance obtenues pour les 6 lectures correspondantes au thermomètre à mercure. Ceci nous a permis de tracer la courbe de réponse de la thermistance à partir des 6 valeurs de résistance effectuées. On peut éviter ces longues mesures d'étalonnage de la résistance de platine, si l'on dispose d'une résistance de platine sélectionnée pour répondre à l'exigence de précision désirée. Il est possible, en effet, de se procurer un élément étalonné au $\frac{1}{2}, \frac{1}{3}$ ou $\frac{1}{4}$ de la norme DIN 73760 autour d'une température choisie.

4. Mesures de régulation. - Nous avons procédé à des essais de stabilité et de reproductibilité de la température affichée. Nous avons effectué les mesures de résistance au pont de Kelvin commercial (ESI 300 PVB)

\section{TABLEAU I}

\begin{tabular}{|c|c|c|c|}
\hline & Thermo & re II & $\begin{array}{l}\text { Différence de } \\
\text { température }\end{array}$ \\
\hline${ }^{\circ} \mathrm{C}$ & 20.9 .74 & ${ }^{\circ} \mathrm{C}$ & ${ }^{\circ} \mathrm{C}$ \\
\hline 一 & 一 & - & - \\
\hline 26,90 & $55,15 \Omega$ & 26,95 & 0,05 \\
\hline 27,55 & 55,26 & 27,55 & 0 \\
\hline 28,10 & 55,37 & 28,15 & 0,05 \\
\hline 28,70 & 55,49 & 28,75 & 0,05 \\
\hline 29,25 & 55,60 & 29,30 & 0,05 \\
\hline 29,90 & 55,72 & 29,95 & 0,05 \\
\hline 30,50 & 55,85 & 30,55 & 0,05 \\
\hline 31,20 & 55,97 & 31,25 & 0,05 \\
\hline 31,80 & 56,10 & 31,80 & 0 \\
\hline 32,50 & 56,24 & 32,55 & 0,05 \\
\hline 33,20 & 56,37 & 33,25 & 0,05 \\
\hline
\end{tabular}

à $\pm 0,01 \Omega$. A cette précision pour des affichages identiques au potentiomètre et le même thermomètre de platine, à un mois d'intervalle, on n'observe pas de variation de température dépassant la précision de l'étalonnage.

Avec un autre thermomètre de platine (étalonné), l'écart le plus grand que nous ayons observé entre les deux mesures est de $0,05^{\circ} \mathrm{C}$.

Chacune de ces mesures a été effectuée après un temps suffisant pour s'assurer de l'équilibre thermique.

Le tableau I résume les résultats obtenus.

Pour connaître la stabilité de la régulation en fonction du temps, nous avons affiché une température pendant plusieurs heures; nous avons constaté une variation autour du point choisi de $\pm 0,02{ }^{\circ} \mathrm{C}$.

5. Conclusion. - La reproductibilité des températures affichées, et la stabilité des conditions de diffusion dans la cellule de mesure placée dans un tel thermostat, nous ont permis d'entreprendre et de mener à bien une étude expérimentale approfondie sur le mouvement Brownien d'une sphère dans un fluide avec mise en évidence d'un comportement non-markovien de celui-ci (J. P. Boon [5]).

Remerciements. - Nous tenons à remercier M. le Professeur G. Sylin pour les conseils éclairés qu'il nous a donnés pour cette réalisation ainsi que MM. Legros et Poty du Service de M. le Professeur Thomaes qui ont mis à notre disposition l'appareillage nécessaire à l'étalonnage des thermomètres. Nos remerciements s'adressent aussi à $\mathrm{M}$. A. Noblet pour ce qui concerne les discussions sur les circuits électroniques et à MM. J. De Saeger et L. Tisseghem pour l'exécution mécanique du thermostat.

\section{Bibliographie}

[1] Baudoux, P., Mesures électriques éd. P. U. B., 1964.

[2] Damaye, R., Amplificateurs opérationnels éd. Radio, 1972.

[3] Scotr, D. W., Experimental thermodynamics, Vol. I : Calorimetry of non reacting systems, éd. J. P. McCullough (1968).
[4] Pièces détachées professionnelles, EP 1501 (2), M. B. L. E. 15] Boon, J. P., Boulller, A., soumis à Phys. Rev. Lett. (mars 1975). 